\title{
Artrodese tibiotalocalcaneana minimamente invasiva com haste intramedular retrógrada bloqueada - Relato de três casos*
}

\section{Minimally Invasive Tibiotalocalcaneal Arthrodesis with Blocked Retrograde Intramedullary Nail - Report of Three Cases}

\author{
${ }^{1}$ Grupo de Cirurgia do Pé e Tornozelo, Serviço de Ortopedia, Hospital Santa \\ Izabel, Santa Casa de Misericórdia da Bahia, Salvador, BA, Brasil \\ 2 Programa de Residência Médica em Ortopedia e Traumatologia, \\ Serviço de Ortopedia, Hospital Santa Izabel, Santa Casa de \\ Misericórdia da Bahia, Salvador, BA, Brasil \\ ${ }^{3}$ Grupo de Oncologia Ortopédica, Hospital Santa Izabel, Santa Casa \\ de Misericórdia da Bahia, Salvador, BA, Brasil \\ Rev Bras Ortop
}

Fernando Delmonte Moreira ${ }^{1}$ ] Jorge Eduardo de Schoucair Jambeiro ${ }^{10}$

Antero Tavares Cordeiro Neto ${ }^{10}$ José Augusto Oliveira ${ }^{10}$ Felipe Fernandes Leão ${ }^{2(0)}$ Alex Guedes ${ }^{3(0)}$

\author{
Endereço para correspondência Alex Guedes, PhD, Grupo de \\ Oncologia Ortopédica, Hospital Santa Izabel, Santa Casa de \\ Misericórdia da Bahia, Rua Marechal Floriano 212, apt. ${ }^{\circ}$ 401, \\ Canela, Salvador, BA, 40110-010, Brasil \\ (e-mail: alexguedes2003@yahoo.com.br).
}

\author{
Resumo \\ Palavras-chave \\ - articulação do \\ tornozelo \\ - artrodese \\ - osteoartrite \\ - procedimentos \\ cirúrgicos \\ minimamente \\ invasivos \\ - procedimentos \\ cirúrgicos operatórios \\ - tornozelo
}

\begin{abstract}
A osteoartrite do tornozelo (OAT) está associada a quadro álgico e limitação funcional variável, demandando tratamento clínico e eventual indicação cirúrgica quando as medidas conservadoras são inefetivas - a artrodese tem sido o procedimento de escolha, por reduzir a dor, restaurar o alinhamento articular e tornar o segmento estável, preservando a marcha. O presente estudo relata 3 casos ( 3 tornozelos) de pacientes do sexo masculino, com entre 49 e 63 anos de idade, portadores de OAT secundária, American Orthopaedic Foot and Ankle Society Ankle-Hindfoot Scale (AOFAS AHS, na sigla em inglês) pré-operatória de 27 a 39 pontos, tratados mediante artrodese tibiotalocalcaneana minimamente invasiva utilizando haste intramedular retrógrada bloqueada. A permanência hospitalar foi de 1 dia, e os pacientes foram autorizados para carga imediata com órteses removíveis para deambulação, conforme tolerado. O tratamento fisioterápico, introduzido desde o internamento, foi mantido, priorizando-se treino de marcha, ganho de força e propriocepção. Foi realizado
\end{abstract}

Trabalho desenvolvido nos Grupos de Cirurgia do Pé e Tornozelo e Oncologia Ortopédica, Hospital Santa Izabel, Santa Casa de Misericórdia da Bahia, Salvador, BA, Brasil.

recebido

22 de Setembro de 2020

aceito

15 de Janeiro de 2021
DOI https://doi.org/ 10.1055/s-0041-1731356. ISSN 0102-3616. (c) 2021. Sociedade Brasileira de Ortopedia e Traumatologia. All rights reserved.

This is an open access article published by Thieme under the terms of the Creative Commons Attribution-NonDerivative-NonCommercial-License, permitting copying and reproduction so long as the original work is given appropriate credit. Contents may not be used for commercial purposes, or adapted, remixed, transformed or built upon. (https://creativecommons.org/ licenses/by-nc-nd/4.0/)

Thieme Revinter Publicações Ltda., Rua do Matoso 170, Rio de Janeiro, RJ, CEP 20270-135, Brazil 


\begin{abstract}

\section{Keywords}

- ankle joint

- arthrodesis

- osteoarthritis

- minimally invasive surgical procedures

- surgical procedures, operative

- ankle

Ankle osteoarthritis (AOA) is associated with pain and variable functional limitation, demanding clinical treatment and possible surgical indication when conservative measures are ineffective - arthrodesis has been the procedure of choice, because it reduces pain, restores joint alignment and makes the segment stable, preserving gait. The present study reports 3 cases ( 3 ankles) of male patients between 49 and 63 years old, with secondary AOA, preoperative American Orthopaedic Foot and Ankle Society Ankle-Hindfoot Scale (AOFAS AHS) of 27 to 39 points, treated by minimally invasive tibiotalocalcaneal arthrodesis using blocked retrograde intramedullary nail. Hospital stay was of 1 day, and the patients were authorized for immediate loading with removable ambulation orthotics, as tolerated. The physical therapy treatment, introduced since hospitalization, was maintained, prioritizing gait training, strength gain, and proprioception. Clinical and radiographic follow-up was performed at weeks 1, 2, 6, 12 and 24. After evidence of consolidation (between the $6^{\text {th }}$ and $10^{\text {th }}$ weeks), the orthotics were removed. One patient complained of pain in the immediate postoperative period and, at the end of the $1^{\text {st }}$ year, only one patient presented pain during rehabilitation, which was completely resolved with analgesics. Currently, the patients do not present complaints, returning to activities without restrictions - one of them, to the practice of soccer and rappelling. The postoperative AOFAS AHS was from 68 to 86 points.
\end{abstract}

acompanhamento clínico e radiográfico nas semanas 1, 2, 6, 12 e 24. Após evidências de consolidação (entre a $6^{\text {a }}$ e a $10^{\text {a }}$ semanas), as órteses foram retiradas. Um paciente queixou-se de dor no pós-operatório imediato e, ao final do $1^{\circ}$ ano, apenas 1 paciente apresentou dor durante a reabilitação, resolvida completamente com analgésicos. Atualmente, os pacientes não apresentam queixas, retornando às atividades sem restrições - um deles, à prática de futebol e rapel. A AOFAS AHS pós-operatória foi de 68 a 86 pontos.

\section{Introdução}

A osteoartrite do tornozelo (OAT) primária é rara, sendo comum a forma secundária ${ }^{1}$ a lesões traumáticas, Charcot, artrite reumatoide e necrose avascular., ${ }^{2,3}$

Há diversas opções de tratamento para a OAT, do manejo clínico ao cirúrgico, quando medidas conservadoras não surtem efeito - as principais opções de tratamento cruento incluem a artrodese e as artroplastias de substituição e de distração. ${ }^{4}$

A artrodese tem sido o procedimento de escolha, por reduzir a dor, restaurar o alinhamento e estabilizar o segmento, preservando a marcha.

A artrodese do tornozelo pode ser realizada mediante utilização de diversos tipos de implante e diferentes vias de acesso, utilizando ou não enxertos ou substitutos ósseos. ${ }^{2-4}$

A artrodese tibiotalocalcaneana (ATTC) minimamente invasiva, mediante haste intramedular retrógrada bloqueada (HIMRB) tem sido indicada pelas vantagens biomecânicas (carga compartilhada, maior rigidez à flexão, compressão dinâmica e estabilidade rotacional) e biológicas (grande área de contato ósseo, procedimento minimamente invasivo, cruentização articular que produz "calda" osteocartilaginosa com potencial hematopoiético). ${ }^{3,5}$
O objetivo do presente estudo é relatar os casos de três portadores de OAT secundária (três tornozelos) submetidos à ATTC minimamente invasiva, utilizando HIMRB.

\section{Descrição Dos Casos}

Três pacientes ( 3 tornozelos) portadores de OAT secundária, atendidos em nossa instituição, foram tratados mediante ATTC minimamente invasiva utilizando HIMRB, após falhas nas medidas conservadoras (casos 1 e 2 ) e falência de artrodese (caso 3) (-Figura 1), no ano de 2017.

Todos eram do sexo masculino, com entre 49 e 63 anos, com dor e limitação funcional variável. A American Orthopaedic Foot and Ankle Society Ankle-Hindfoot Scale (AOFAS AHS, na sigla em inglês) $)^{6}$ era de 27 a 39 pontos (-Tabela 1).

Os pacientes foram posicionados em decúbito dorsal em mesa cirúrgica radiotransparente, sob sedação, bloqueio e antibioticoprofilaxia, sem isquemia ou tração. As articulações foram acessadas mediante três portais, um subtalar e dois tibiotalares (anterolateral e anteromedial), previamente marcados sob fluoroscopia com agulha descartável $40 \times 12 \mathrm{~mm}$. Foram feitas incisões de 1,0 a $1,5 \mathrm{~cm}$ nas marcações e dissecção romba até as cápsulas articulares, ampliando a área de trabalho (-Figura 2). Foi introduzida uma fresa cônica motorizada de $4,3 \mathrm{~mm}$ para cruentização 

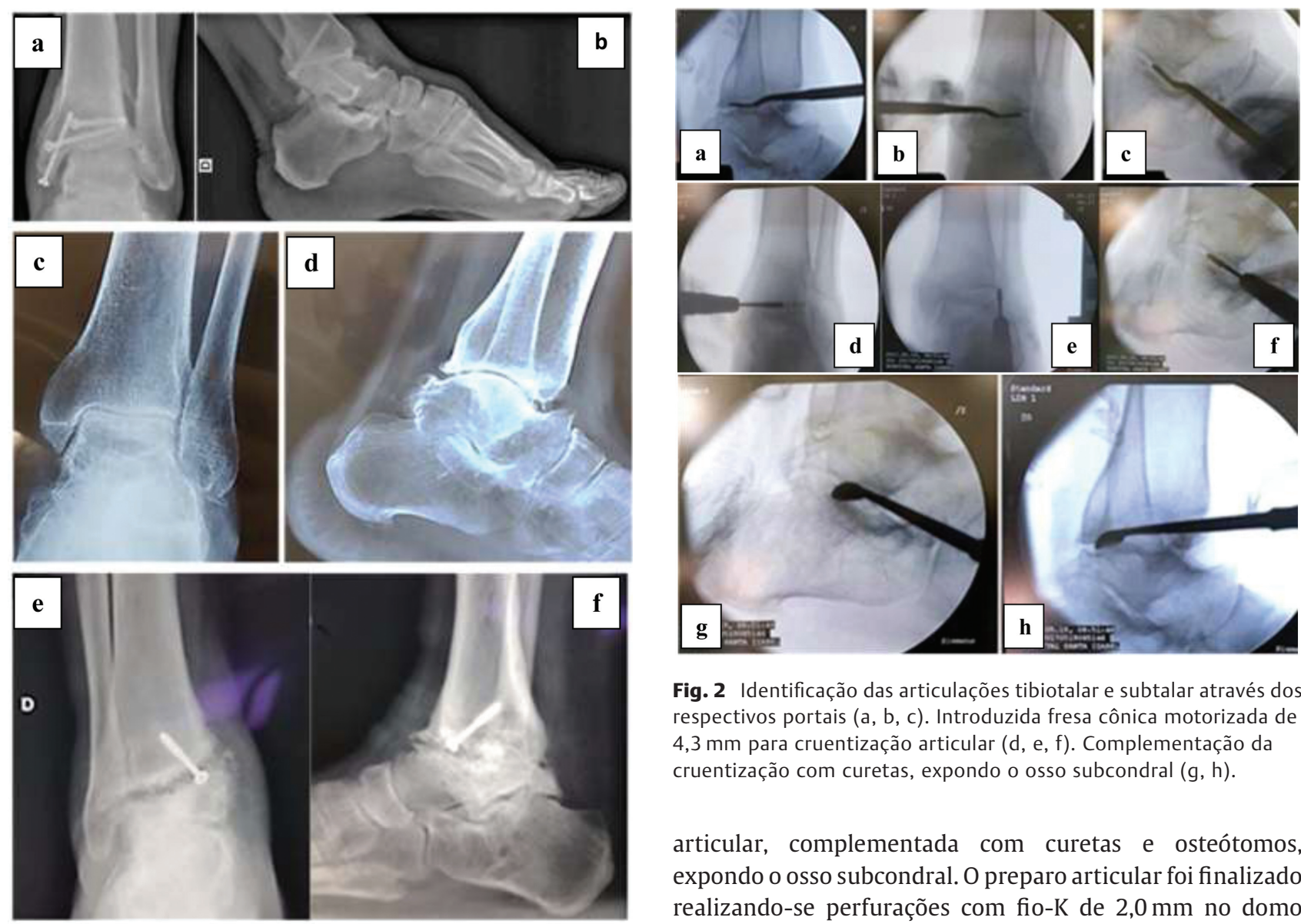

Fig. 2 Identificação das articulações tibiotalar e subtalar através dos respectivos portais (a, b, c). Introduzida fresa cônica motorizada de 4,3 mm para cruentização articular (d, e, f). Complementação da cruentização com curetas, expondo o osso subcondral $(\mathrm{g}, \mathrm{h})$.

articular, complementada com curetas e osteótomos, expondo o osso subcondral. 0 preparo articular foi finalizado realizando-se perfurações com fio-K de $2,0 \mathrm{~mm}$ no domo talar e na superfície articular tibial (-Figura 2). Foi realizada fixação com HIMRB, da maneira tradicional. Foram feitas suturas de pele e curativo compressivo. Todos os procedimentos evoluíram sem intercorrências.

Fig. 1 Aspecto radiográfico pré-operatório: caso 1 - sequela de fratu do pilão tibial (a, b); caso 2 - instabilidade crônica do tornozelo (c, d); e caso 3 - falha na artrodese tibiotalar (e, f).

Tabela 1 Descrição dos achados clínicos e American Orthopaedic Foot and Ankle Society Ankle-Hindfoot Scale pré e pósoperatórios e complicações

\begin{tabular}{|l|l|l|l|}
\hline & Caso 1 & Caso 2 & Paciente 3 \\
\hline Gênero & Masculino & Masculino & Masculino \\
\hline Idade (Anos) & 49 & 61 & 63 \\
\hline Queixa Principal & Dor & Dor & Dor \\
\hline Lado & Esquerdo & Esquerdo & Direito \\
\hline Arco de Movimento & $5^{\circ}$ & $25^{\circ}$ & $10^{\circ}$ \\
\hline Deformidade & Valgo & Neutro & Valgo \\
\hline Diagnóstico & $\begin{array}{l}\text { Sequela de fratura } \\
\text { do pilão tibial }\end{array}$ & $\begin{array}{l}\text { Instabilidade crônica } \\
\text { do tornozelo }\end{array}$ & $\begin{array}{l}\text { Falha na artrodese } \\
\text { tibiotalar }\end{array}$ \\
\hline Início de Carga (Semanas) & 1 & 1 & 1 \\
\hline Tempo de Consolidação (Semanas) & 10 & 6 & 8 \\
\hline AOFAS Pré-operatório & 39 & 33 & 27 \\
\hline AOFAS Pós-operatório & 68 & 72 & 86 \\
\hline Complicação Precoce & Não & Dor, resolvida com analgésicos & Não \\
\hline Complicação Tardia & Não & Não & $\begin{array}{l}\text { Dor, resolvida com } \\
\text { analgésicos }\end{array}$ \\
\hline
\end{tabular}

Abreviação: AOFAS, American Orthopaedic Foot and Ankle Society Ankle-Hindfoot Scale 

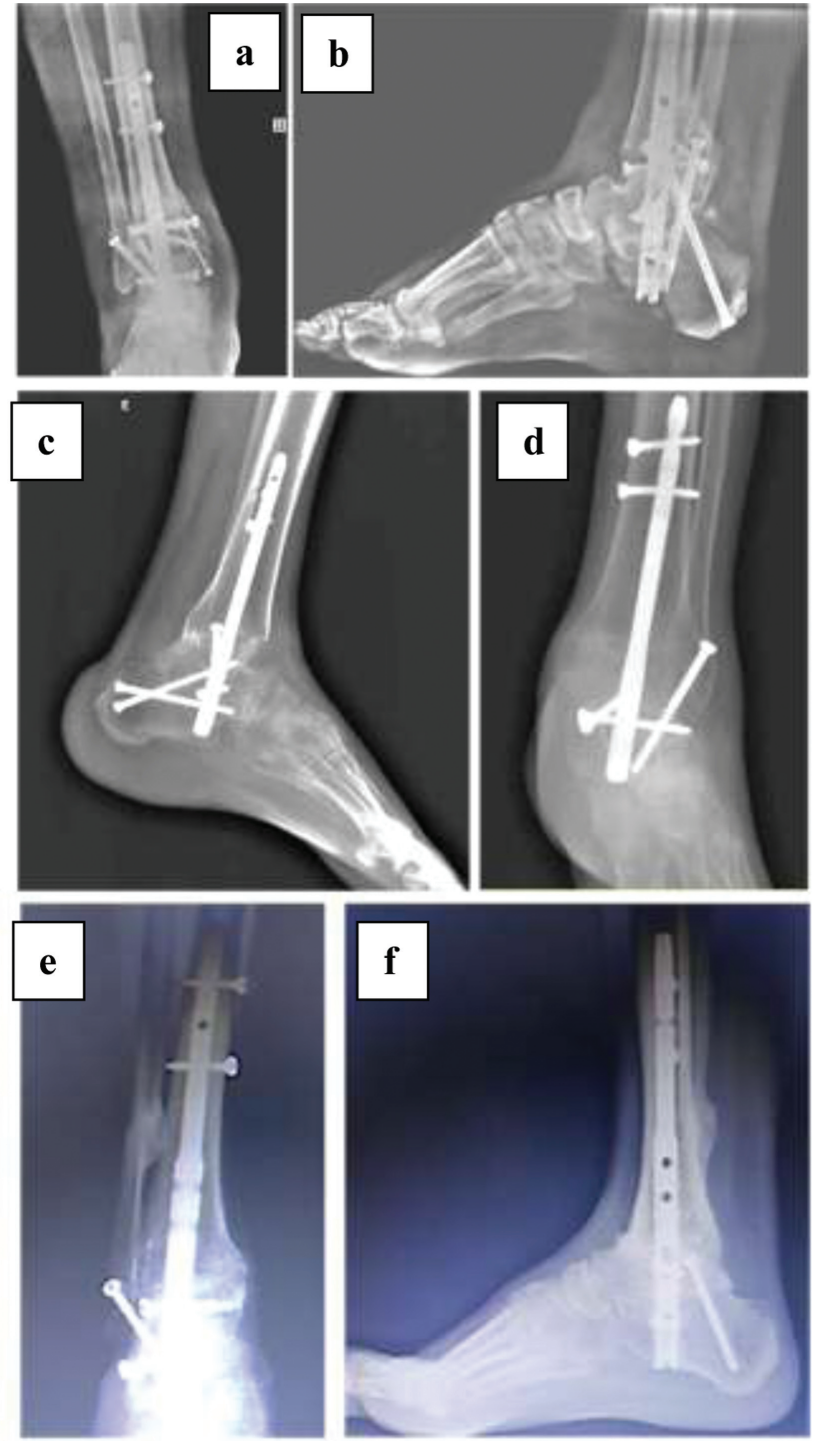

Fig. 3 Aspecto radiográfico no pós-operatório tardio dos casos 1 (a, b), 2 (c, d) e 3 (e, f).

Alta no $1^{\circ}$ dia pós-operatório (PO). Foi iniciada carga imediata, conforme tolerado, utilizando órteses removíveis para deambulação. Os pontos foram retirados no $15^{\circ}$ dia PO.

0 tratamento fisioterápico foi introduzido no internamento e seguiu ambulatorialmente, priorizando-se o treino de marcha, ganho de força e propriocepção.

Foi feito acompanhamento clínico e radiográfico ( - Figura 3) nas semanas 1, 2, 6, 12 e 24. Após evidências de consolidação, entre a $6^{\underline{a}}$ e $10^{\underline{a}}$ semanas, as órteses foram retiradas (-Figura 4).

Um paciente queixou-se de dor no PO imediato, resolvida com analgésicos. Ao final do $1^{\circ}$ ano, apenas 1 paciente apresentou dor na reabilitação, resolvida completamente com analgésicos. Atualmente ( $3^{\circ}$ ano $\mathrm{PO}$ ) os pacientes não apresentam queixas.

Todos os pacientes voltaram às atividades sem restrições um deles, à prática desportiva (futebol e rapel). A AOFAS AHS $^{6}$ pós-operatória foi de 68 a 86 pontos (-Tabela $\mathbf{1}$ ).

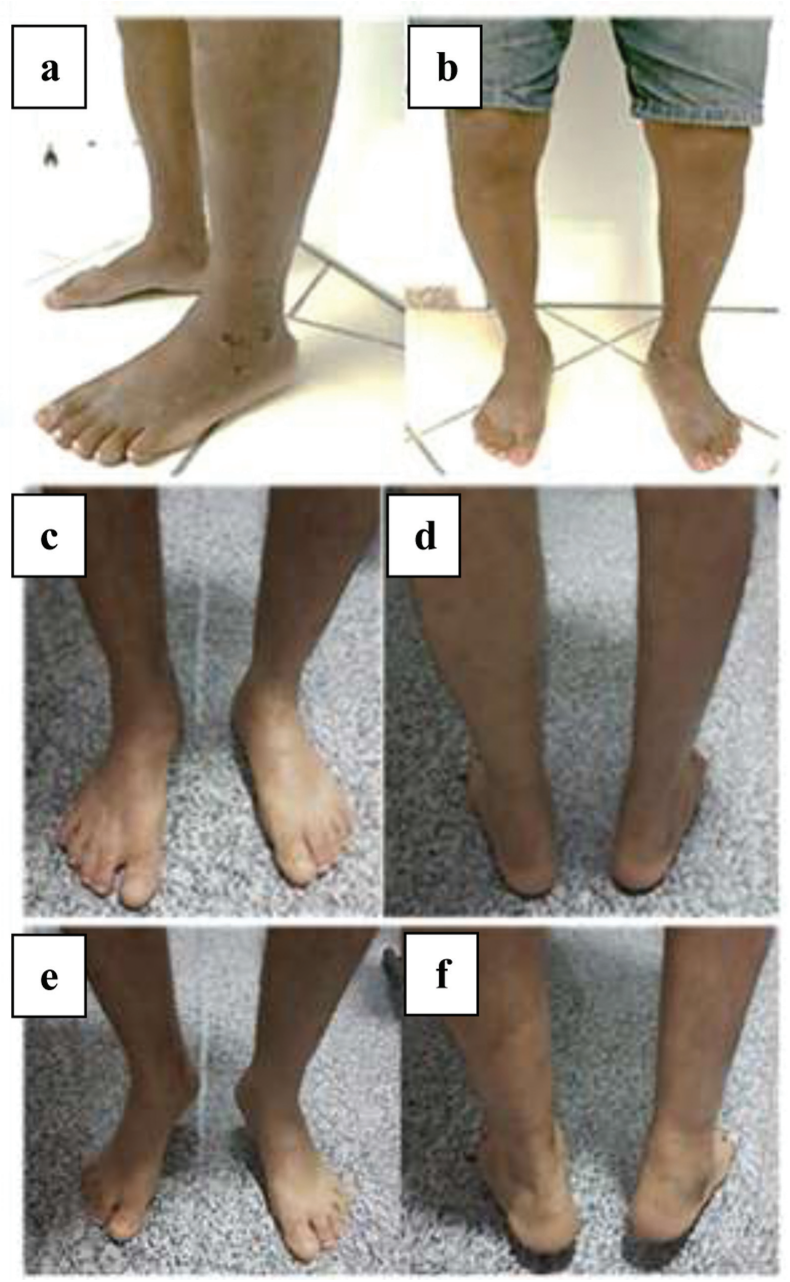

Fig. 4 Aspecto pós-operatório do caso 2 (a, b) e caso 3 (c, d, e, f).

\section{Discussão}

A ATTC aberta está bem estabelecida no tratamento da OAT, independente da causa, e constitui uma excelente alternativa no tratamento de pacientes com condições pré-operatórias ruins (baixo estoque ósseo, desalinhamento do retropé ou histórico de múltiplos procedimentos).

Entretanto, apesar de prover ampla exposição, a ATTC aberta demanda maior permanência e está sujeita a complicações como infecção, deiscência e pseudartrose, potencializadas por comorbidades, frequentemente presentes nos pacientes submetidos a este procedimento. ${ }^{3}$

Em estudo com 20 pacientes submetidos à ATTC aberta com HIMRB por OAT, Charcot e deformidades, observou-se união em $80 \%$ das articulações tibiotalares e subtalares e em $20 \%$ das tibiocalcaneanas. A AOFAS AHS progrediu de $54.20 \pm 15.71$ para $76.0 \pm 11.63(p<0.001)$. A permanência média foi de 6,7 dias. Houve alto índice de complicações, principalmente infecções (35\%), culminando com uma amputação abaixo do joelho. ${ }^{7}$

Rammelt et al. ${ }^{8}$ avaliaram a ATTC aberta com HIMRB em 38 pacientes, por não união, OAT, deformidade, Charcot e falha pósartroplastia. Os autores descreveram alinhamento adequado em $92 \%$ e fusão em $84 \%$ dos pacientes. A permanência média foi de 
8,4 dias. Foi identificado um risco de $24 \%$ para pelo menos uma complicação pós-operatória, sendo não união a mais comum, seguida por problemas com implantes e infecção.

Em estudo retrospectivo, ${ }^{9} 29$ pacientes com deformidade foram submetidos a ATTC aberta com HIMRB, obtendo-se fusão articular em 96,6\%. Houve aumento médio da AOFAS AHS de 29,7 para 74,3 ( $\mathrm{p}<0.01)$. Como complicações, três casos de stress tibial, três de neuropraxia e três de infecção.

Diante do potencial para complicações, alguns autores advogam abordagens minimamente invasivas, dentre elas a ATTC percutânea mediante HIMRB. ${ }^{3,5}$

Biz et al. $^{5}$ apresentaram 28 pacientes tratados com ATTC mediante HIMRB percutânea, a maioria por OAT pós-traumática, e observaram $100 \%$ de consolidação e $92,85 \%$ de alinhamento plantígrado e estável. Como complicações, um caso de protrusão de parafuso e um de retardo de consolidação, com dor associada.

Uma revisão sistemática com metanálise ${ }^{2}$ incluiu 8 pacientes tratados mediante ATTC aberta e 15 por via artroscópica. Três pacientes submetidos a ATTC aberta e quatro a artroscópica apresentavam úlcera plantar. As taxas de fusão foram similares (75 versus 67\%; $p=0.679$ ). Complicações ocorreram em $63 \%$ das ATTC abertas (80\% infecções) e em 33\% das artroscópicas (100\% não união). A presença de úlcera não influenciou na gênese de infecção nas ATTC abertas (67 versus 60\%); entretanto, houve aumento significativo de não união nas artroscópicas (75 versus $18 \% ; p=0.039$ ). Pacientes sem úlcera apresentaram taxa de união de $80 \%$ para ambas as metodologias.

Apresentamos três casos de OAT tratados mediante ATTC percutânea com HIMRB. 0 tempo de permanência (1 dia) foi consideravelmente menor do que o da literatura para a abordagem aberta ( 3 a 8 dias). 0 tempo de consolidação (6 a 10 semanas) foi inferior aos procedimentos cruentos (12 semanas). A AOFAS AHS ${ }^{6}$ pré-operatória de 27 a 39 evoluiu para 68 a 86, achado corroborado pela literatura para a ATTC com HIMRB, ${ }^{2,3,5,7-10}$ sendo observada apenas dor precoce ( 1 paciente) e dor tardia ( 1 paciente), após 1 ano, as quais foram resolvidas, sem outras complicações até o momento.

\section{Suporte Financeiro}

Não houve suporte financeiro de fontes públicas, comerciais, ou sem fins lucrativos.

\section{Conflito de Interesses}

Os autores declaram não haver conflito de interesses.

\section{Referências}

1 Saltzman CL, Salamon ML, Blanchard GM, et al. Epidemiology of ankle arthritis: report of a consecutive series of 639 patients from a tertiary orthopaedic center. Iowa Orthop J 2005;25:44-46

2 Baumbach SF, Massen FK, Hörterer S, et al. Comparison of arthroscopic to open tibiotalocalcaneal arthrodesis in high-risk patients. Foot Ankle Surg 2019;25(06):804-811

3 Vilà y Rico J, Rodriguez-Martin J, Parra-Sanchez G, Marti LopezAmor C. Arthroscopic tibiotalocalcaneal arthrodesis with locked retrograde compression nail. J Foot Ankle Surg 2013;52(04): 523-528

4 Coughlin MJ, Nery C, Baumfeld D, Jastifer J. Artrodese tibiotársica compressiva com o uso de placa bloqueada lateral. Rev Bras Ortop 2012;47(05):611-615

5 Biz C, Hoxhaj B, Aldegheri R, Iacobellis C. Minimally invasive surgery for tibiotalocalcaneal arthrodesis using a retrograde intramedullary nail: preliminary results of an innovative modified technique. J Foot Ankle Surg 2016;55(06):1130-1138

6 Rodrigues RC, Masiero D, Mizusaki JM, et al. Translation, cultural adaptation and validity of the american orthopaedic foot and ankle society (AOFAS) ankle-hindfoot scale. Acta Ortop Bras 2008; 16(02):107-111

7 Lee BH, Fang C, Kunnasegaran R, Thevendran G. Tibiotalocalcaneal arthrodesis with the hindfoot arthrodesis nail: a prospective consecutive series from a single institution. J Foot Ankle Surg 2018;57(01):23-30

8 Rammelt S, Pyrc J, Agren PH, et al. Tibiotalocalcaneal fusion using the hindfoot arthrodesis nail: a multicenter study. Foot Ankle Int 2013;34(09):1245-1255

9 Brodsky JW, Verschae G, Tenenbaum S. Surgical correction of severe deformity of the ankle and hindfoot by arthrodesis using a compressing retrograde intramedullary nail. Foot Ankle Int 2014; 35(04):360-367

10 Thomas AE, Guyver PM, Taylor JM, Czipri M, Talbot NJ, Sharpe IT. Tibiotalocalcaneal arthrodesis with a compressive retrograde nail: A retrospective study of 59 nails. Foot Ankle Surg 2015; 21(03):202-205 